\title{
Suboptimal Optics: Vision Problems as Scars of Evolutionary History
}

\author{
Steven Novella
}

Published online: 25 October 2008

(C) Springer Science + Business Media, LLC 2008

\begin{abstract}
The human eye is an excellent example of suboptimal bottom-up design resulting from the constraints of evolutionary historical contingency. The resulting suboptimal optics manifests in a number of medical ophthalmological disorders.
\end{abstract}

Keywords Bottom-up process · Evolution .

Suboptimal optics $\cdot$ Eye evolution

Evolution is the scientific theory that the various species of life on earth arose through branching descent, change, and speciation over time. This is a decidedly bottom-up process of design with populations adapting to their immediate needs using the raw material that happens to be available to them. This raw material includes their existing biology in all its facets, including the variation within the population. Evolution is therefore unguided, unplanned, messy, quirky, and historically contingent.

This evolutionary perspective should help us make sense of the quirky details of biology and anatomy we find in nature, such as the anatomy of the human eye. I will take a decidedly medical perspective - I am a physician and physicians are familiar with all the ways in which biology can go wrong. Diseases and medical problems often arise from the quirkiness of bottom-up design, so I will examine various aspects of eye anatomy and discuss how they lead to functional problems that could easily have been avoided by a more rational top-down design.

Figure 1 is the human eye, cut away to show the internal structures, including the retina that line the inside of the globe of the eye and is the tissue layer that reacts to light.

\footnotetext{
S. Novella $(\bowtie)$

Yale University School of Medicine,

New Haven, USA

e-mail: steven.novella@yale.edu
}

Figure 2 is a microscopic view of all the layers that make up the retina. In this figure, light would enter from the bottom and travel up to the receptors (rods and cones) near the top.

\section{The "Backwards" Retina}

The most obvious design flaw of the retina is that the cellular layers are backwards. Light has to travel through multiple layers in order to get to the rods and cones that act as the photoreceptors. There is no functional reason for this arrangement - it is purely quirky and contingent.

Even in a healthy and normally functioning eye, this arrangement causes problems. Because the nerve fibers coming from the rods and cones need to come together as the optic nerve, which then has to travel back to the brain, there needs to be a hole in the retina through which the optic nerve can travel. This hole creates a blind spot in each eye. Our brains compensate for this blind spot so that we normally do not perceive it - but it is there.

From a practical point of view, this is a minor compromise to visual function, but it is completely unnecessary. If the rods and cones were simply turned around so that their cell bodies and axons were behind them (oriented to the direction of light), then there would be no need for a blind spot at all.

Some creationists have tried to counter this argument by saying the layers need to be arranged this way. For example, Dr. George Marshall in an interview for answersingenesis.org says:

"The light-detecting structures within photoreceptor cells are located in the stack of discs. These discs are being continually replaced by the formation of new ones at the cell body end of the stack, thereby pushing 


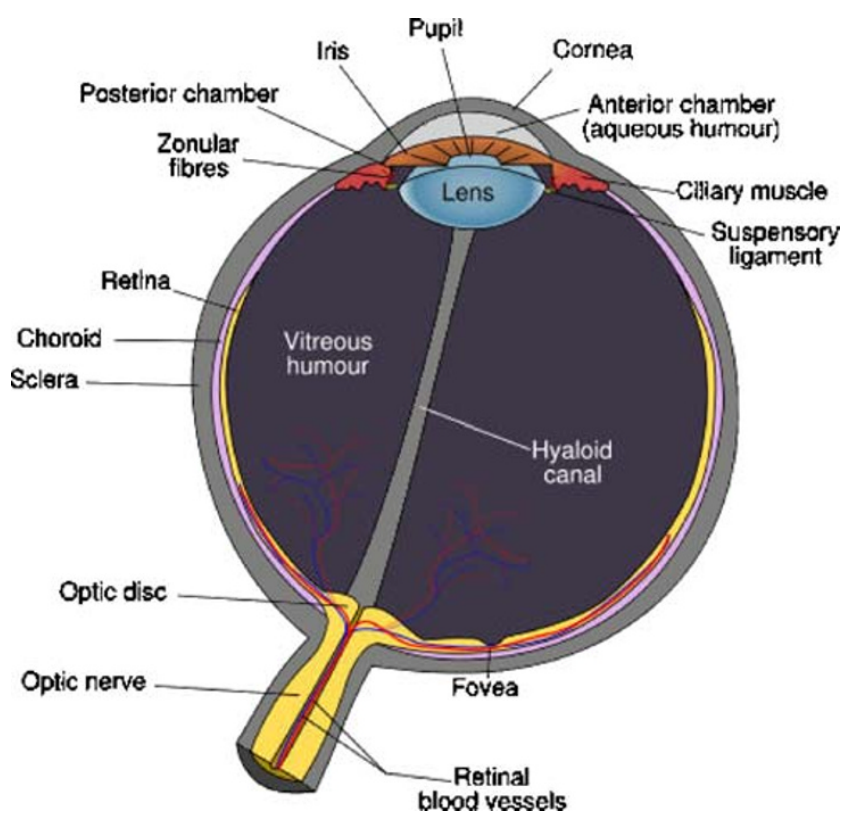

Fig. 1 The human eye

older discs down the stack. Those discs at the other end of the stack are 'swallowed' by a single layer of retinal pigment epithelial (RPE) cells. RPE cells are highly active, and for this they need a very large blood supply - the choroid. Unlike the retina, which is virtually transparent, the choroid is virtually opaque, because of the vast numbers of red blood cells within it. For the retina to be wired the way that Professor Richard Dawkins suggested, would require the choroid to come between the photoreceptor cells and the light, for RPE cells must be kept in intimate contact with both the choroid and photoreceptor to perform their job. Anybody who has had the misfortune of a hemorrhage in front of the retina will testify as to how well red blood cells block out the light." (Casson 2008)

This answer is clearly false because the evolutionary line that led to modern cephalopods, like the squid, evolved eyes independently from the vertebrate line leading to humans (an example of convergent evolution). The squid eye has the photoreceptor layer at the top of the retina, unlike the vertebrate configuration which has it at the bottom (see Fig. 3; Cavallerano 1992). It is therefore demonstrably possible for this better configuration to work. Evolutionary contingency reaches different results from different histories. Top-down design, however, offers no explanation for the use of a superior design in one case and a suboptimal design in another.

It just so happens that the primitive vertebrates that first developed a primitive eye were small and transparent, and it is postulated that the arrangement of the retina therefore did not matter. Either way, once the anatomical choice was made, it constrained all of future vertebrate eye evolution.

Marshall's answer also does not make internal sense because it assumes that certain elements of the current retinal design are necessary and unchangeable-which is only the case in a contingent system, but is certainly not the case in a system designed to its current form without the constraint of history. For example, the rods and cones could have been designed so that the photoreceptor discs are produced at the top (meaning the layer closest to the direction of light), with older ones moving backward toward the bottom of the cells where they are absorbed. Below this absorption layer could be the blood vessels, and the axons from the rods and cones could also leave from the bottom of the rods and cones through this opaque absorption layer (the RPE).

Even worse than the backward arrangement of the rods and cones is the fact that the blood vessels that feed the retinal sit on top of the retina-between the light source and the receptive layer. A more logical arrangement would be to have the blood vessels feed the retina from behind, so that they do not get in the way. In healthy eyes, the blood vessels do not cause any perceptible problem (but they are also partly responsible for the blind spot), but they do limit the total amount of light reaching the rods and cones. The real problem is that they are vulnerable to various diseases.

About $80 \%$ of diabetics who have had diabetes for 10 years will develop diabetic retinopathy (Klein et al. 1994). In response to chronic ischemia (relative lack of oxygen), the retina will produce chemical signals that tell the blood vessels to proliferate to increase the blood supply. Because the blood vessels are above the retina, they increasingly get in the way, obscuring vision. At present,

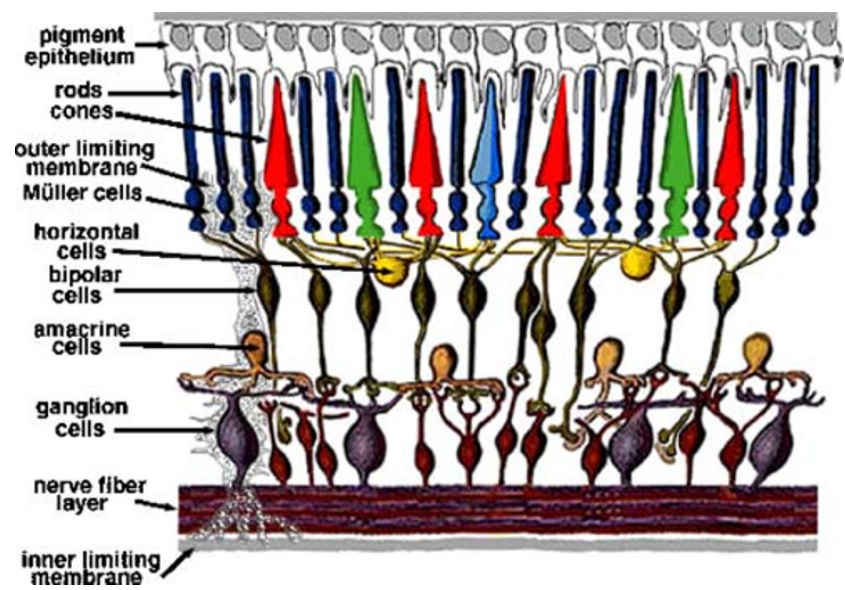

Fig. 2 Microscopic view of all the layers that make up the retina 
Fig. 3 Configuration of the cephalopod and vertebrate eye

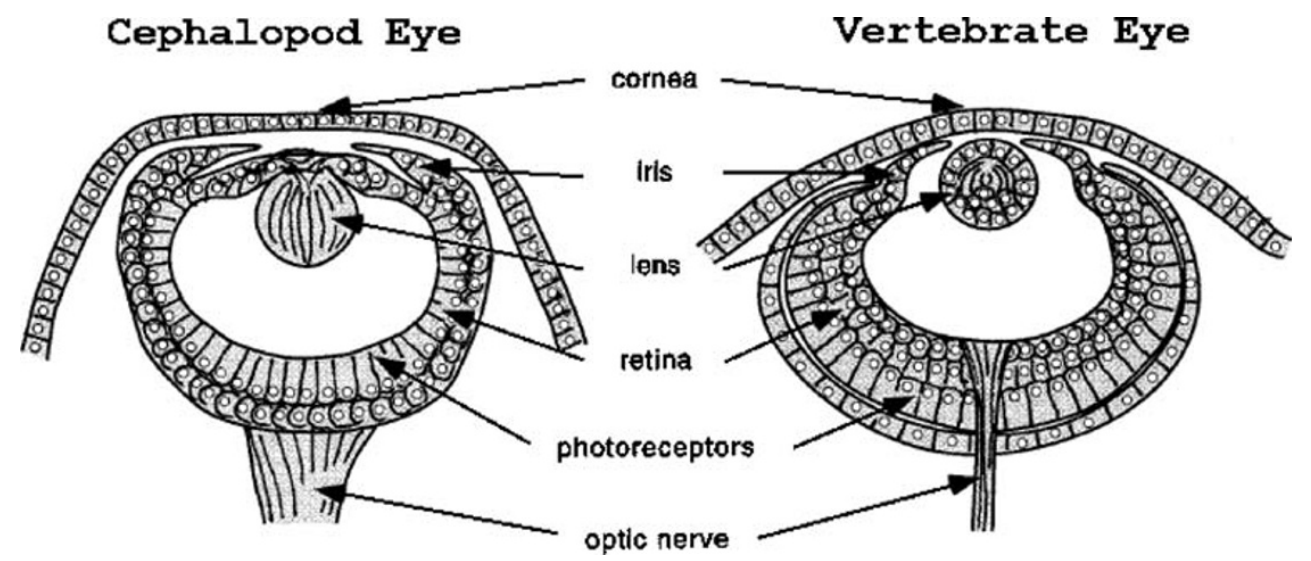

the primary treatment of diabetic retinopathy is to use a laser to burn some of the blood vessels and decrease their proliferation.

Having the blood vessels in front of the retina also means that even a small retinal hemorrhage can significantly impair vision.

And finally, any edema or inflammation that occurs within the cell layers in front of the rods and cones will likewise impair vision. All of this could have been avoided or minimized were the rods and cones placed in the most superficial layer of the retinal, rather than buried at the bottom.

\section{Detached Retina}

Yet another consequence of the "backward" arrangement of the retina is the susceptibility to detached retina. This occurs when the photoreceptor layer becomes detached from the pigment epithelium beneath (Litzinger and Del Rio-Tsonis 2002). The subsequent loss of nourishment causes blindness in the detached part of the retina. One cause of retinal detachment is minor trauma to the retina that allows fluid to leak and build up between the retina and the pigment epithelium, thus separating the layers. But the more common cause is simple aging, that results in subtle changes to the shape of the globe of the eye and loss of the elasticity of the vitreous humor-the gel that fills the globe.

Again, the cephalopod eye does not suffer from retinal detachment because the axons from the photoreceptors anchor them to the layers beneath.

\section{Macular Degeneration}

The macula is that part of the retina that has the densest concentration of rods and cones for detailed vision. Within the macula is a smaller area called the fovea which contains only cones and has the highest density of these receptors. The very existence of the macula, however, is a partial fix for the "backward" arrangement of retinal layers with the nerve and blood vessels between the receptors and the direction of light. This limits the density of rods and cones, and so the partial fix is to have one small area cleared of nerves and blood vessels where rods and cones can be denser. However, if the human retina were designed like that of the squid and other cephalopods, this would not be necessary.

The dependence of the human eye on the macular for sharp vision creates a vulnerability, for any problem with that small area will have a dramatic effect on visual acuity. The rest of the retina will not be able to adequately compensate for the loss or compromise of the macula because the density of rods and cones is just too diffuse.

Macular degeneration is the most common cause of blindness. Although the cause is often unknown, its severe effects on vision are a consequence of the need for a macula as a partial fix for the poor retinal design.

\section{Angle Closure Glaucoma}

Glaucoma results from increased pressure inside the eye. Between the iris and the cornea is a space called the anterior chamber which is filled with the aqueous humor-a clear liquid that is constantly made and drained from this chamber. If the drainage is blocked or slowed, then the fluid will back up, increasing the pressure inside the eye. This pressure can damage the optic nerve, resulting in blindness. In acute angle closure glaucoma, blindness can occur within hours and it is therefore an emergency.

The draining channels, called the trabecular meshwork, are at the angle between the iris and the cornea. When this angle gets too narrow because the iris is pushing up against 
the meshwork, then drainage can be blocked. This is another example of suboptimal design - the drainage is unnecessarily precarious and susceptible to blockage, leading to rapid loss of vision.

Through the quirkiness of genetics, some populations are more susceptible to angle closure glaucoma than others because they have an inherently narrower angle to start with. The most susceptible populations are Asians and Inuit. There is speculation that the narrow angle may have been a thermoregulatory adaptation to colder climates - a compromise exacerbating an already existing design weakness (Marshall 1996).

\section{The Extraocular Muscles}

The arrangement of the extraocular muscles-the muscles that move the eyes - is also difficult to explain without appealing to evolutionary contingency. There are more muscles than are minimally necessary and yet there is no functional redundancy. In order to move a sphere in any direction, only three muscles would be necessary, evenly spaced like the legs of a tripod. The human eye has six-the superior, inferior, lateral, and medial rectus, and the superior and inferior oblique. And yet, despite the extra three muscles, the loss of function of any one muscle causes an impairment of eye movement and results in double vision or displaced vision. A more frugal design with only three muscles would be more efficient and less prone to malfunction, as there are fewer components to break down.

If the eye were to be designed with more than the minimal three muscles, then it would make sense to arrange the muscles so that the loss of one or even more would not impair eye movement.

Fossil evidence suggests that primitive jawed fishes had seven extraocular muscles (Whitnall 1911). Some modern vertebrates retain this configuration, such as dogs that have a 7th extraocular muscle, the retractor bulbi, not found in humans, although there is a report of an incidence of the retractor bulbi occurring in a human, a likely recitavistic trait (Young 2008).

The configuration of cranial nerve control of the extraocular muscles also makes no design sense. The lateral rectus is controlled by cranial nerve VI (abducens), the superior oblique by cranial nerve IV (trochlear), and the rest by cranial nerve III (oculomotor). There is no functional advantage to this particular arrangement; it is an accident of evolution. Having three cranial nerves responsible for eye muscles multiplies the opportunity for failure of any one, and again there is no redundancy as a hedge against malfunction.
But the most suboptimal aspect of cranial nerve innervation of the extraocular muscles is the abducens, cranial nerve VI. The abducens takes an unnecessarily long path from the brainstem, through the skull, and to the lateral rectus. There is no design reason for this long path-it too is an accident of evolution. It makes the abducens particularly vulnerable to injury or stretching, and for this reason, abducens palsy is the most common extraocular muscle weakness. The trochlear nerve (cranial nerve IV to the superior oblique) also takes an unusual pathway-it exits the brainstem heading toward the back of the brain, which is the wrong direction. It must then swing around and head toward the eyes. As with the abducens nerve, this unnecessarily long pathway increases the potential for malfunction.

\section{Susceptibility to Disease}

The human eye is susceptible to a number of other diseases and dysfunctions that do not result from any obvious "design flaw" but for which there is no particular protection either. This is a weaker line of evidence for evolution because there is no clear flaw to point to, simply the lack of design elements that could have protected the eye from problems. For example, if the shape of the eye and cornea are not within certain narrow parameters then the image will not focus on the retina, leading to nearsightedness or farsightedness. It is not difficult to imagine plausible mechanisms to correct these so-called refractive errors-such as mechanisms for distorting the shape of the cornea or the globe of the eye. This could be done with muscles or by increasing or decreasing the fluid behind the cornea and within the globe.

Cataracts form when the proteins in the lens begin to clump together, causing the lens to become cloudy and obscuring vision. A protein design that does not allow for this clumping, or a repair mechanism to replace clumped proteins, could render the lens immune to cataracts. The lens also tends to become stiff with age, reducing its ability to focus near objects onto the retina. As a result the near point, the closest point that a person can bring into focus, moves slowly farther away, causing what is called presbyopia. Most people, when they get into their $40 \mathrm{~s}$ or $50 \mathrm{~s}$, need to use reading glasses for this reason.

Again, by itself, this is not a compelling line of evidence for evolution. But since evolution has been adequately established through multiple other independent lines of evidence, it can provide a helpful perspective by which such susceptibilities to eye malfunction can be best understood. 


\section{Conclusion}

Biologist Frank Zindler said, "As an organ developed via the opportunistic twists and turns of evolutionary processes, the human eye is explainable. As an organ designed and created by an infinitely wise deity, the human eye is inexcusable." (Zindler 1986).

Zindler is essentially referring to the contrast between a bottom-up process of design and a top-down process. Bottom-up systems, such as evolution, are unplanned, quirky, and are constrained by their history. Top-down systems do not have such constraints.

Stephen J. Gould pointed out that the difference will not be found in the complexity and perfection of anatomy and physiology - for both top-down and bottomup origins are compatible with biological forms that are well adapted to their current functions.

Evolution will be evident, rather, in the imperfections, suboptimal design, quirky anatomy, and other manifestations of contingent evolutionary history. The medical malfunctions of the human eye provide excellent examples of this principle.

\section{References}

Casson RJ. Anterior chamber depth and primary angle-closure glaucoma: an evolutionary perspective. Clin Experiment Ophthalmol. 2008;36(1):70-7. doi:10.1111/j.1442-9071.2008.01672.x.

Cavallerano AA. Retinal detachment. Optom Clin. 1992;2(3):25-70.

Klein R, Klein BE, Moss SE, Cruickshanks KJ. The Wisconsin Epidemiologic Study of diabetic retinopathy. XIV. Ten-year incidence and progression of diabetic retinopathy. Arch Ophthalmol. 1994;112(9):1217-28.

Litzinger T, Del Rio-Tsonis K. Eye anatomy. Encyclopedia of life science; 2002, April 15.

Marshall G. An eye for creation. http://www.answersingenesis.org/ creation/v18/i4/eye.asp September; 1996.

Whitnall SE. An instance of the retractor bulbi muscle in man. J Anat Physiol. 1911;46(Pt 1):36-40.

Young GC. Number and arrangement of extraocular muscles in primitive gnathostomes: evidence from extinct placoderm fishes. Biol Lett. 2008;4(1):23.

Zindler F. Half a wing and no prayer. The Probing Mind; 1986, April. 